\title{
Oxidative stress and total antioxidant status in term newborns and their mothers
}

\author{
Maria Wilinska', Maria K. Borszewska-Kornacka², Tomasz Niemiec ${ }^{3}$, Grzegorz Jakiel ${ }^{4}$ \\ ${ }^{1}$ Department of Neonatology, Medical Centre of Postgraduate Education, Warsaw, Poland \\ ${ }^{2}$ Department of Neonatology and Neonatal Intensive Care, Medical University, Warsaw, Poland \\ ${ }^{3}$ Department of Animal Nutrition and Biotechnology, Warsaw University of Life Sciences, Warsaw, Poland \\ ${ }^{4}$ I Department of Obstetrics and Gynecology, Medical Centre of Postgraduate Education, Warsaw, Poland
}

Wilinska M, Borszewska-Kornacka MK, Niemiec T, Jakiel G. Oxidative stress and total antioxidant status in term newborns and their mothers. Ann Agric Environ Med. 2015; 22(4): 736-740. doi: 10.5604/12321966.1185786

\begin{abstract}
The peripartum period and delivery are considered critical for maintaining a balance between the production of free oxygen radicals and functional incompetence of the antioxidant system of a foetus and a neonate.

Objective. The aim of the study was to evaluate the oxidoreductive state of mothers and their newborns immediately after delivery and in the first few days after birth.

Method. Eighty-five mothers and their healthy, term newborns were included into the prospective study. Total antioxidant capacity (TAC) and thiobarbituric acid reactive substances (TBARS) in maternal placenta and milk, as well as blood and urine of the neonates on the first and third day after birth, were examined.

Results. Elevated levels of TBARS were found in maternal placenta and neonatal blood on the third day after birth, but low concentrations were observed in maternal milk. On the contrary, total antioxidant status (TAS) showed a declining tendency in neonatal blood and an increasing tendency in breast milk. Markers of oxidative stress and antioxidant capacity were independent of the mode of delivery.

Conclusions. Values of oxidative stress markers in mothers and newborns immediately after birth were elevated and increased further during the first few days of neonatal life. Breast milk was the only nutritional substance with high antioxidant activity. At the same time, TBARS levels in breast milk decreased, which might indicate its protective role in reducing oxidative stress in newborns.
\end{abstract}

\section{Key words}

oxidative stress, pregnancy, labor, neonate, breast milk

\section{INTRODUCTION}

Reactive oxygen species (ROS), such as superoxide radical anion $\mathrm{O}_{2}$, hydrogen peroxide $\mathrm{H}_{2} \mathrm{O}_{2}$ and hydroxyl radical are unstable chemical metabolites of oxygen, formed by numerous chemical and metabolic processes occurring within a living organism. A growing number of studies report the participation of ROS in the development of infant diseases. Newborns are particularly prone to oxidative damage induced by ROS. Oxidative stress affects various organs, mostly the lung epithelium, retinal and brain blood vessels. Free oxygen radicals are produced in cell membranes and structures such as peroxisomes, mitochondria, endoplasmic reticulum, protein cell membranes and soluble enzymes (haemoglobin, xanthine oxygenase). Sensitivity of cells and tissues results from their rapid growth and development, as well as weak antioxidant protection. Proteins, lipids and DNA are damaged. Significant structural alterations take place and lead to cell death by apoptosis or necrosis [1].

The antioxidant status of newborns reveals low concentrations of glutathione peroxidase, superoxide dismutase, $ß$-carotene, riboflavin, $\alpha$-proteinase, vitamin E, selenium, copper, zinc, transferrin and other plasmatic factors [2].

Address for correspondence: Maria Wilińska, Department of Neonatology, Medical Centre of Postgraduate Education, Warsaw, Czerniakowska 231, 00-436 Warsaw, Poland

E-mail:wilinska.maria@gmail.com

Received: 06 July 2013; accepted: 18 January 2014

\section{OBJECTIVE}

1) to assess the oxidative state and reduction potential in mothers and their newborns;

2) to characterize the dynamics of oxidative stress change during the first days after birth.

\section{MATERIALS AND METHOD}

A prospective observational clinical study was conducted between 1 August 2010 - 30 April 2011 at the Neonatology Clinic and First Clinic of Gynaecology and Obstetrics, Professor W. Orłowski Independent Public Clinical Hospital, Postgraduate Medical Education Centre (PMEC), Warsaw. The study was approved by the PMEC Ethics Committee. The study group comprised 85 newborns, born at term, and their mothers. Demographic details of the study population are presented in Table 1.

The study protocol included the following information: maternal condition during pregnancy, course of the delivery (mode of delivery, premature rupture of the membranes and the character of the amniotic fluid), prenatal steroid intake, chorioamnionitis, and drug administration during labour.

Demographic characteristics of the neonates included their weight and gestational age. Apgar score, $\mathrm{pH}$ and S-100 protein concentration, as well as lactate levels in the umbilical cord blood were used to evaluate the condition of the infant. Oxidative stress and antioxidant capacity were determined 
in the biological materials (placenta, colostrum, umbilical cord blood, neonatal urine and maternal blood) obtained from the mothers and their children:

- on the first day - maternal placenta: $3-5 \mathrm{~g}$ from the area of the umbilical cord attachment, colostrum $(3 \mathrm{ml})$ and umbilical cord blood ( $1 \mathrm{ml})$;

- on the third day - neonatal blood collected for general diagnostic tests $(1 \mathrm{ml})$, neonatal urine $(5 \mathrm{ml})$ and breast milk (3ml).

Blood samples were drawn and allowed to clot, then centrifuged, divided into 2 portions and frozen at $-80^{\circ} \mathrm{C}$. Urine and human milk were frozen at $-80^{\circ} \mathrm{C}$.

Oxidative stress was measured with TBARS (thiobarbituric acid reactive substances) assay. Reactions of end-products of lipid peroxidation (malondialdehyde) with thiobarbituric acid represent the state of lipids found mostly in the structures of cell membranes. Their elevated levels suggest increased lipid peroxidation or impaired repair mechanisms [3]. S-100 protein concentration was measured in the placenta, umbilical cord blood, neonatal urine and breast milk.

The activity of TAS was examined by Randox kit (NX2332). Lactate concentrations and the $\mathrm{pH}$ of umbilical cord blood were assessed in the umbilical arterial blood and performed with the use of the Roche OMNI 5 analyzer.

Statistical analysis. The sample size was estimated at $\mathrm{a}=0.05$ type I error level. The $\boldsymbol{t}$ test with repeated measures was used to verify the primary study hypothesis - comparison of the levels of the studied markers (S-100 protein, TAS, TBARS) on days 1 and 3 after birth. Statistical analysis was then extended with the mixed-model analysis of variance (F test).

The study assumed to prove an association between the studied parameters and S-100 protein concentration. For this purpose, Pearson's R correlation between S-100 protein level and the remaining parameters was calculated. Additionally, $t$ test for independent samples was performed to determine whether the level of all examined parameters was dependent on the following factors: mode of delivery, gender, infection in pregnancy, lowered Apgar score.

\section{RESULTS}

The study was conducted in a group of 85 healthy newborns born at term. Table 1 presents the demographic characteristics of the group and basic data on the course of pregnancy and the labor.

The condition of the newborns was assessed by means of the Apgar score. All newborns included into the study scored $\geq 9$ points at 1 and 5 minutes of life. Foetal biochemical markers, $\mathrm{pH}$ and lactate levels in the umbilical cord blood were within the normal range in all infants.

S-100 protein level in the umbilical blood cord after Caesarean section was higher than after vaginal delivery. In both cases, the concentration decreased on the third day of neonatal life.

The oxidative stress intensity in the placenta (Tab. 3) was higher in cases of instrumental labour, Apgar scores $<10$, and maternal infections during pregnancy. None of the differences were statistically significant.

Analysis and comparison of oxidative stress levels in various biological materials revealed that TBARS values were
Table 1. Complications of pregnancy

\begin{tabular}{|c|c|c|}
\hline Parameter & & No. of children \\
\hline \multirow{3}{*}{ Gestational age [hbd] } & $36-37$ & 9 \\
\hline & $38-40$ & 64 \\
\hline & $41-42$ & 12 \\
\hline \multirow{3}{*}{ Weight [g] } & $<2500$ & 1 \\
\hline & $2500-4000$ & 77 \\
\hline & $>4000$ & 7 \\
\hline \multirow{2}{*}{ Gender } & male & 53 \\
\hline & female & 32 \\
\hline \multirow{3}{*}{ Mode of delivery } & VD & 60 \\
\hline & $\mathrm{CC}$ & 24 \\
\hline & Forceps & 1 \\
\hline \multirow{2}{*}{ Infections in pregnancy* } & No & 67 \\
\hline & Yes & 18 \\
\hline \multirow[t]{5}{*}{ Course of pregnancy } & Diabetes & 6 \\
\hline & Hypertension & 6 \\
\hline & Antenatal steroid therapy & 3 \\
\hline & $\mathrm{PROM}>18 \mathrm{~h}$ & 1 \\
\hline & Chorioamnionitis & 0 \\
\hline \multirow{2}{*}{ Apgar score } & $1 \min 9.73$ & \\
\hline & $5 \min 9.94$ & \\
\hline umbilical cord arterial blood & $\begin{array}{l}\mathrm{pH} 7.339 \\
\text { lactates }[\mathrm{mg} / \mathrm{dL}] 42.59\end{array}$ & \\
\hline
\end{tabular}

* infection of genital tract $(n=14)$, urinary tract $(n=3)$ or respiratory tract $(n=4)$

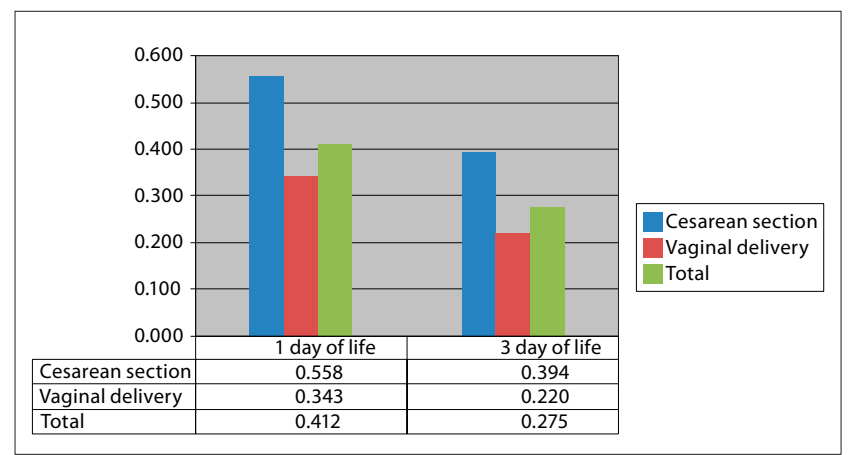

Figure 1. S-100 protein concentration in umbilical cord blood on days 1 and 3 of life.

Table 2. Oxidative stress [TBARS] - placenta

\begin{tabular}{|c|c|c|c|}
\hline & & $\begin{array}{c}\text { TBARS mean } \\
(\mathrm{nmol} / \mathrm{ml})\end{array}$ & $\mathrm{p}$ \\
\hline \multirow{2}{*}{ Mode of delivery } & VD & 64.82 & \multirow{2}{*}{0.134} \\
\hline & CC & 74.58 & \\
\hline \multirow{2}{*}{ Apgar (1 min.) } & $=10$ & 66.73 & \multirow{2}{*}{0.535} \\
\hline & $<10$ & 71.58 & \\
\hline \multirow{2}{*}{ Infection in pregnancy } & no & 66.56 & \multirow{2}{*}{0.522} \\
\hline & yes & 71.12 & \\
\hline \multirow{2}{*}{ Gender } & male & 65.73 & \multirow{2}{*}{0.491} \\
\hline & female & 70.57 & \\
\hline
\end{tabular}

higher in neonatal blood than breast milk immediately after birth, and continued to grow until the third day after birth. The increase was statistically significant. TBARS in breast 


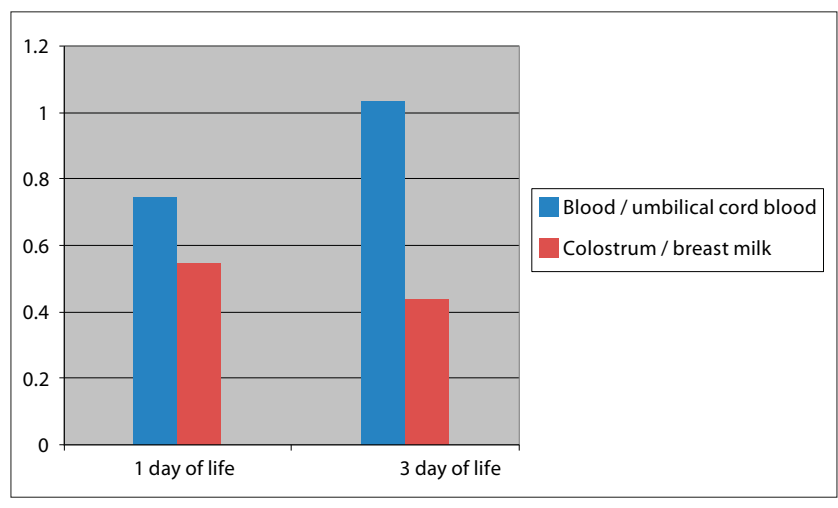

Figure 2. Oxidative stress TBARS $[\mathrm{nmol} / \mathrm{ml}]$ in neonatal blood and breast milk on days 1 and 3 of life

Table 3. TBARS - neonatal urine on day 3 of life

\begin{tabular}{|c|c|c|c|}
\hline & & $\begin{array}{c}\text { Mean (nmol/mg } \\
\text { creatinine) }\end{array}$ & $\mathrm{p}$ \\
\hline \multirow{2}{*}{ Mode of delivery } & VD & 13.56 & \multirow{2}{*}{0.208} \\
\hline & $\mathrm{CC}$ & 16.22 & \\
\hline \multirow{2}{*}{ Apgar (1 min.) } & $=10$ & 14.41 & \multirow{2}{*}{0.825} \\
\hline & $<10$ & 13.84 & \\
\hline \multirow{2}{*}{ Infection in pregnancy } & no & 14.51 & \multirow{2}{*}{0.691} \\
\hline & yes & 13.56 & \\
\hline \multirow{2}{*}{ Gender } & male & 12.52 & \multirow{2}{*}{0.045} \\
\hline & female & 17.26 & \\
\hline
\end{tabular}

milk were not only lower at the initial level, but continued to statistically significantly decrease $(\mathrm{p}<0.005)$.

The activity of TBARS was clearly visible in urine, with statistically significant differences observed in females, to their disadvantage. TBARS concentration was independent of the mode of delivery, Apgar score or maternal infections during pregnancy.

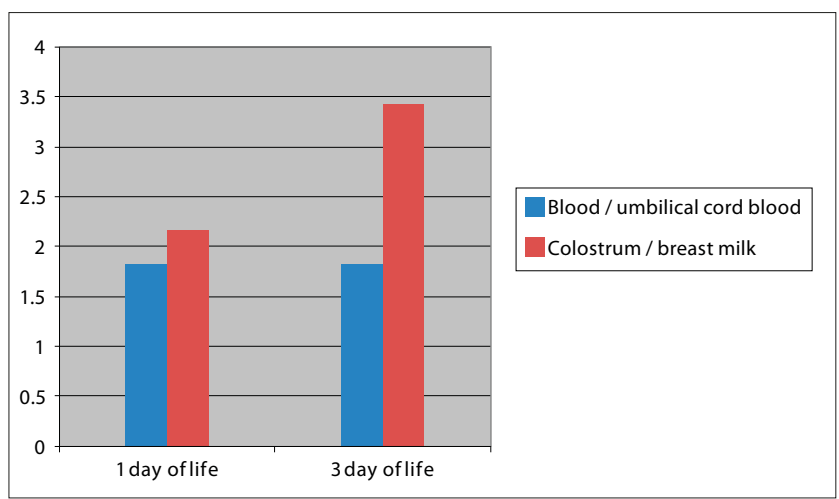

Figure 3. Antioxidant capacity TAS $[\mathrm{mmol} / \mathrm{L}]$ in neonatal blood and breast milk on days 1 and 3 of life

Total antioxidant capacity in infant blood remained stable during the first three days of neonatal life. TAS concentration visibly increased in breast milk and reached statistical significance on the third day after birth.

A mixed-design analysis of variance demonstrated no influence of the delivery mode, lower Apgar score $(<9)$ or genital, respiratory and urinary tract maternal infections during pregnancy on the TBARS, TAS and S-100 protein levels. The influence of maternal complications on the oxidative stress parameters was not analyzed due to a small number of cases with diabetes and hypertension.

Analysis of the correlation between S-100 protein and TAS and TBARS, separately for the first and third days after birth, did not reveal significant dependence, apart from a significant correlation between S-100 protein concentration in the umbilical cord blood and TBARS in the colostrum on the first day after birth $(r=376 ; p<0.05)$. In the absence of clear trends in the remaining parameters, the result might have been accidental.

$\boldsymbol{t}$ test for independent samples. Correlation between lactate levels and $\mathrm{pH}$ of the umbilical cord blood, TAS, S-100 protein and TBARS and such factors as the mode of delivery, gender, maternal infections during pregnancy (urinary, genital or respiratory tract), and lower Apgar score $(<10)$ at 1 minute of life, was analyzed.

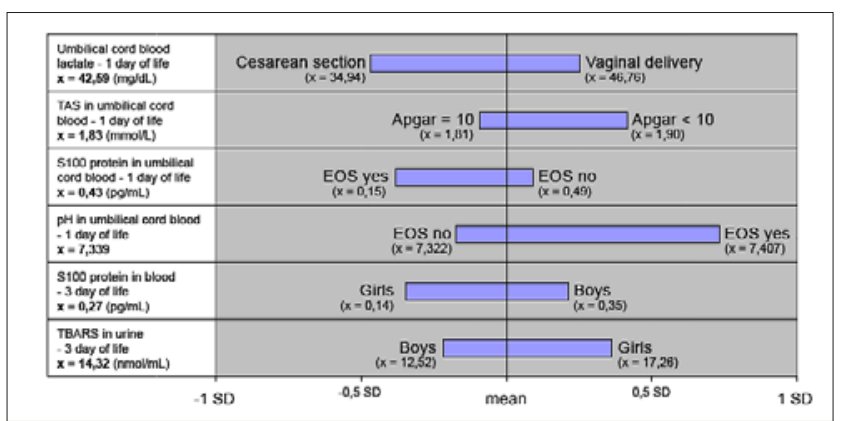

Figure 4. $t$ test for independent, standardized samples, statistical significance at $p<0.05$.

$\mathrm{SD}$ - standard deviation; $\mathrm{x}$ - mean

There were a few cases where the differences reached or were on the borderline of statistical significance. No specific trend or direction was noted; therefore, it is difficult to draw unambiguous conclusions. Despite statistical significance, the results might have been accidental.

Caesarean section proved to be the reason of elevated TAS and TBARS in neonatal blood on the third day after birth when compared to vaginal delivery. Infants with early onset sepsis had higher TAS levels than healthy neonates. Female neonates demonstrated higher TAS values, but the differences are not statistically significant.

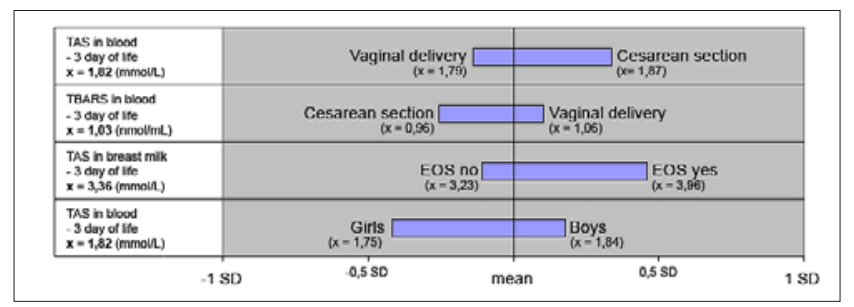

Figure 5. $t$ test for independent, standardized samples

SD - standard deviation; $\mathrm{x}$ - mean.

\section{DISCUSSION}

Higher oxygen demand due to increased rate of metabolism during pregnancy may stimulate the production of ROS. Lipid peroxidation typically increases during pregnancy. The placenta is rich in polyunsaturated fatty acids which, due to 
increased metabolism, undergo the processes of peroxidation easier and the lipid peroxidation products are released into the circulation.

The obtained results confirmed that TBARS were severalfold higher in placenta tissue samples than in other biological samples.

Although antioxidant activity during physiological pregnancy is genetically determined, antioxidant concentration may be insufficient to balance high production of free radicals. Numerous pregnancy-related complications (foetal death, intrauterine growth restriction, pre-eclampsia) are the result of insufficient maternal antioxidant protection to balance high production of free oxygen radicals and lipid peroxidation in the placenta [4].

Nevertheless, oxidative enzyme activity in the peripheral blood of the mother is not always an indicator of oxidative stress in complicated pregnancy. Researchers from the Department of Pathology of Pregnancy and Gynaecology at the Institute of Polish Mother's Health Centre in Lodz, Poland, reported a decreased concentration of catalase in a pregnant woman during instrumental delivery due to the threat of eclampsia. However, this did not negatively influence the condition of the mother and the neonate [5].

Saker et al. documented a significant increase of oxidative stress parameters and protection against antioxidant activity in newborns with intrauterine growth restriction [6].

Oxidative stress during the foetal period programmes the metabolism of the neonate either directly, through gene expression modulation, or indirectly, through the influence of ROS on lipids and proteins in the critical window of development [7].

Gestational diabetes is an example of a disease where oxidative stress plays a key causative role in metabolic disturbances and their consequences. Rajdl et al. found a statistically significant difference in the TBARS levels of diabetic mothers and their children in comparison to healthy mothers, suggesting an exceptional free radical activity expressed as increased lipid peroxidation [8]. At the same time, antioxidant protection, manifested by antioxidant enzyme activity, was significantly lower.

The disturbances also affect foetal metabolism. Neonates are born with elevated oxidative stress parameters and decreased antioxidant capacity, compared to healthy newborns from physiological pregnancies. This phenomenon concerns mothers treated with insulin, as well as oral hypoglycaemic agents and diet. Intensification of complications in neonates has been found to correlate with the degree of hyperglycaemia in their mothers. Since there were only 6 mothers with gestational diabetes in the presented study group, considerable differences in the group sizes did not allow for statistically significant conclusions to be drawn.

Delivery is associated with considerable oxidative stress and the maturity of the newborn, as well as the circumstances of the delivery, influence its extent. Some authors claim that elective Caesarean section significantly reduces antioxidant capacity in preterm and term infants $[6,9]$.

Oxidative stress may last also after the delivery. The presented study demonstrated that prooxidative activity continues to grow in neonatal blood for at least three days after birth. These results are similar to those of Perrone et al. [10]. The presence of oxidative stress markers may also be demonstrated in the umbilical cord blood, indicating the risk of foetal exposure to ROS during intrauterine life.
Preterm newborns are at high risk of complications resulting from oxidative stress. Various investigations proved that prematurely born children have limited possibilities of antioxidative activity in response to hyperoxia. Defence against free radicals increases shortly before the delivery - a $150 \%$ increase is observed in the last $15 \%$ of the pregnancy time $[4,11]$ - in particular the defence dependent on the activity of the following enzymes: glutathione, superoxide dismutases, catalases. Prenatal steroid administration also significantly intensifies that activity [12].

In the face of these threats, studies on the influence of factors protecting neonates from the harmful effects of free oxygen radicals seem particularly valuable. Breast milk, especially after preterm delivery, contains high concentration of antioxidant defence factors that are highly valuable for the newborns, especially preterm infants [13].

Perrone et al. demonstrated a beneficial effect of lutein, selectively accumulating in the retina [14]. Gitto et al. indicate the positive influence of even a short-term melatonin therapy on reducing oxidative stress in newborns. Melatonin, a known quencher of various ROS, is secreted by the foetal pineal gland, mostly in the first trimester of pregnancy. The hormone plays an important role in foetal growth and neuroprotective activity. After birth, a fullterm neonate does not produce melatonin for 2-4 months, whereas in a preterm infant the deficiency may last up to 8 months of life. Neurological damage intensifies melatonin deficiency [15].

Lee and Davis reviewed the latest results of studies on attempts to treat tissue damage caused by free radicals [16]. A study by Perrone et al. demonstrated a strong correlation between iron-binding protein concentration and the prevalence of the so-called free radical-related diseases in newborns, i.e. intraventricular haemorrhage, periventricular leucomalacia, patent ductus arteriosus, retinopathy of prematurity and bronchopulmonary dysplasia [10].

Various exogenous (ascorbic acid, tocopherol, carotenoids, flavonoids) and endogenous (proteins, bilirubin, uric acid, cholesterol, glutathione) factors form an antioxidant barrier in blood.

The presented study proved that breast milk is a natural source of antioxidant defence. The antioxidant capacity of breast milk is even higher than that of neonatal blood and increases for at least 3 days after birth. The significance of that phenomenon is especially great in the face of increasing oxidative stress markers in neonatal blood, expressed as TBARS. It is yet another argument in favour of the thesis that breast milk has important, other than nutritional, values for an infant.

Ledo et al. demonstrated that preterm newborns eliminate a greater amount of oxidative stress metabolites in urine than term infants [17]. The extent of the elimination correlates with gestational age and weight.

Higher elimination of oxidative stress markers (8-oxodG and o-tyrosine) is observed also in formula-fed children when compared to breastfed infants, proving that preterm babies are exposed to higher oxidative stress than term neonates, and that breastfeeding reduces that stress more than formula feeding [17].

Increased elimination of oxidative stress markers (8-oxodG) was also noted in women with prolonged vaginal delivery and emergency Caesarean section [18]. The presented results indicate a tendency to accumulate both TAS and TBARS 
markers in blood after Caesarean section, although the indications for that particular procedure were not analyzed.

S-100 protein concentration in neonatal blood on the third day after birth decreases in comparison to the first day. It is noteworthy that, although the statistical significance was not reached, a clear tendency (almost a two-fold decrease on day 3 compared to day 1 after birth) was found. It seems that lack of statistical significance might be the result of considerable difference of standard deviation of both measurements which, in turn, might be the effect of the small sample size or high degree of variance within the studied population.

\section{CONCLUSIONS}

1. Healthy, term newborns demonstrate biochemical features of oxidative stress that persist for at least 3 days after birth.

2. Intensity of oxidative stress is independent of the mode of delivery and maternal infections during pregnancy.

3. Maternal milk contains considerable antioxidative capacity, which might have a protective influence on the breastfed infant.

4. Further studies on larger populations are necessary to confirm the hypothesis about the protective effect of human milk on neonatal oxidative stress.

\section{Acknowledgement}

The study was financed by CMKP Project No. 501-1-13-36-10.

\section{REFERENCES}

1. Marcocchi B, Perrone S Paffetti P, et al. Non-protein-bound iron plasma protein oxidative stress at birth. Pediatr Res. 2005; 5: 1295-1299.

2. Buonocore G, Groenendaal F. Antioxidant strategies. Semin Fetal Neonatal Med. 2007; 12: 287-295.

3. Wang W, Pang CCP, Rogers MS, et al. Lipid peroxidation in cord blood at birth. Am J Obstet Gynecol. 1996; 174: 62-65.
4. Al-Gubory KH, Flower PA, Garrel C The roles of cellular reactive oxygen species, oxidative stress and antioxidants in pregnancy outcomes Int J Biochem Cell Biol. 2010; 42: 1634-1650.

5. Biesiada L, Pietrzak Z, Brocka U, et al. Niektóre wykładniki stresu oksydacyjnego w ciąży powikłanej nadciśnieniem tętniczym i cholestazą. Ginekol Pol.2007; 78: 956-960 (in Polish).

6. Saker M, Mokhtari NS, Merzouk SA, et al. Oxidant and antioxidant status in mothers and their newborns according to birthweight. Eur J Obstet. 2008; 14: 95-99.

7. Luo ZC, Fraser WD, Julien P, et al. Tracing the origins of „fetal origins” of adult diseases: programming by oxidative stress? Med Hypotheses. 2009: 74: 318-332.

8. Rajdl D, Racek J, Steinerova A, et al. Markers of Oxidative Stress in Diabetic Mothers and Their Infants During Delivery. Physiol Res. 2005; 54: 429-436.

9. Georgeson GD, Szony BJ, Streitman K, et al. Antioxidant enzyme activities are decreased in preterm infants and in neonates born via caesarean section. Eur J Obstet Gynecol Reprod Biol. 2002; 103: 136-139.

10. Perrone S, Tataranno ML, Negro S, et al. Early identification of the risk for free radical-related diseases in preterm newborns. Early Hum Dev. 2010; 86: 241-244.

11. Davis JM, Auten RL. Maturation of the antioxidant system and the effects on preterm birth. Sem Fet Neonat Med. 2010; 15: 191-195.

12. Vento M, Aguar M, Escobar J, et al. Antenatal steroids and antioxidant enzyme activity in preterm infants: influence of gender and timing. Antioxid Redox Signal. 2009; 11: 2945-2955.

13. Friel JK, Martin SM, Langdon M, Herzberg GR, Buettner GR. Milk from mothers of both premature and full-term infants provides better antioxidant protection than does infant formula. Pediatr Res. 2002; 51: 612-618.

14. Perrone S, Longini M, Marzocchi B, et al. Effects of lutein on Oxidative Stress in the Term Newborn: A Pilot Study. Neonatology 2010; 97: 36-40.

15. Gitto E, Pellegrino S, Gitto P, et al. Oxidative stress of the newborn in the pre-and postnatal period and the clinical utility of melatonin. J Pineal Res. 2009; 46: 128-139.

16. Lee J, Davis J. Future applications of antioxidants in premature infants. Curr Opin Pediatr. 2011; 22: 161-166.

17. Ledo A, Arduini A, Asensi MA. Human milk enhances antioxidant defenses against hydroxyl radical aggression in preterm infants. Am J Clin Nutr. 2009; 89: 210-215.

18. Chiba T, Omori O, Takahashi K. Correlations between the detection of stress-associated hormone /oxidative stress markers in umbilical cord blood and the physical condition of the mother and neonate. J Obstet Gynaecol Res. 2010; 5: 958-964. 